PROCEEDINGS OF THE

AMERICAN MATHEMATICAL SOCIETY

Volume 128, Number 1, Pages 53-64

S 0002-9939(99)05474-X

Article electronically published on September 9, 1999

\title{
OPTIMAL SIZE ESTIMATES FOR THE INVERSE CONDUCTIVITY PROBLEM WITH ONE MEASUREMENT
}

\author{
G. ALESSANDRINI, E. ROSSET, AND J. K. SEO \\ (Communicated by Lesley M. Sibner)
}

\begin{abstract}
We prove upper and lower estimates on the measure of an inclusion $D$ in a conductor $\Omega$ in terms of one pair of current and potential boundary measurements. The growth rates of such estimates are essentially best possible.
\end{abstract}

\section{INTRODUCTION}

Given a body $\Omega$ whose electrical conductivity is $\sigma \equiv 1$ and a possibly disconnected (unknown) inclusion $D \subset \Omega$, $\operatorname{dist}(D, \partial \Omega) \geq d_{0}$, for some positive number $d_{0}$ of conductivity $\sigma \equiv k, 0<k \neq 1$, the inverse conductivity problem with one measurement consists in determining $D$ by measuring on $\partial \Omega$ the voltage corresponding to one applied current flux. In mathematical terms, if $u$ denotes the electrostatic potential in $\Omega$, one wishes to recover $D$ in the equation

$$
\operatorname{div}\left(\left(1+(k-1) \chi_{D}\right) \nabla u\right)=0 \quad \text { in } \Omega,
$$

by the knowledge of one pair of Cauchy data on $\partial \Omega$

$$
\begin{array}{ll}
\frac{\partial u}{\partial \nu}=\varphi & \text { on } \partial \Omega, \\
u=g & \text { on } \partial \Omega,
\end{array}
$$

where $\nu$ denotes the unit exterior normal to $\partial \Omega$ and $\chi_{D}$ denotes the characteristic function of $D$.

The research on this subject has been mainly devoted to the uniqueness question, that is, whether one pair $\{g, \varphi\}$ uniquely determines $D$. Several partial results have been found $[\mathrm{C}],[\mathrm{BF}],[\mathrm{Al}],[\mathrm{FI}],[\mathrm{IP}],[\mathrm{BFI}],[\mathrm{P}],[\mathrm{BFS}],[\mathrm{AIP}],[\mathrm{AI}],[\mathrm{S}]$. Nonetheless a general uniqueness result is still missing. Recently investigations have been carried out asking whether, rather than the precise shape and location of $D$, one can extract, from the measurements $\{g, \varphi\}$, some reduced kind of information on $D$, such as its size $[\mathrm{AR}],[\mathrm{KSS}]$. Let us also recall the pioneering work by Friedman $[\mathrm{F}]$ and subsequently by Bryan $[\mathrm{Br}]$.

In the present paper we significantly improve upon the results of $[\mathrm{AR}],[\mathrm{KSS}]$ by merging and refining the different techniques of the two papers.

Received by the editors February 11, 1998.

2000 Mathematics Subject Classification. Primary 31B20; Secondary 35R25, 35R30, 35R05.

Key words and phrases. Inverse conductivity problem, size estimates, Muckenhoupt weights.

This research was supported in part by Fondi MURST $40 \%$ and $60 \%$ and by CNR.

(C)1999 American Mathematical Society 
In order to illustrate our main results, let us assume here, for the sake of simplicity, that the conductivity in $D$ is $k>1$. Consider the solution $u_{0}$ to the Neumann problem $(1.1)-(1.2)_{N}$ when $D$ is replaced by the empty set, namely

$$
\Delta u_{0}=0 \quad \text { in } \Omega,
$$

$$
\frac{\partial u_{0}}{\partial \nu}=\varphi \quad \text { on } \partial \Omega
$$

and let $g_{0}=\left.u_{0}\right|_{\partial \Omega}$.

Theorem 2.1 below states that if $D$ satisfies some mild a priori assumption (see (1.7) below), then its measure is comparable with the boundary integral $\delta \mathcal{W}=$ $\int_{\partial \Omega}\left(g_{0}-g\right) \varphi$, that is, we have

$$
C_{1} \frac{\delta \mathcal{W}}{\int_{\partial \Omega} g_{0} \varphi} \leq|D| \leq C_{2} \frac{\delta \mathcal{W}}{\int_{\partial \Omega} g_{0} \varphi}
$$

where $|D|$ denotes the Lebesgue measure of $D$, and $C_{1}, C_{2}$ are estimated in terms of the known data $\Omega, d_{0}, k, \varphi$ and the a priori data on $D$.

Notice that the above integral $\delta \mathcal{W}$ has a very clear physical interpretation. Denoting by $\mathcal{W}=\int_{\Omega}\left(1+(k-1) \chi_{D}\right)|\nabla u|^{2}, \mathcal{W}_{0}=\int_{\Omega}\left|\nabla u_{0}\right|^{2}$ the powers induced by the boundary current $\varphi$ on $\partial \Omega$ when the inclusion $D$ is respectively present and absent, we have by standard integration by parts $\delta \mathcal{W}=\int_{\partial \Omega}\left(g_{0}-g\right) \varphi=\mathcal{W}_{0}-\mathcal{W}$.

An estimate of the type (1.3) was already obtained in [KSS] under restrictive assumptions on $\varphi$. In $[\mathrm{AR}]$ instead, the Dirichlet data are assumed to be prescribed, and letting $u_{0}$ be the solution of $(1.1)^{0}$ with Dirichlet data $g$, the measure of $D$ was evaluated in terms of $\delta \tilde{\mathcal{W}}=\int_{\partial \Omega}\left(\varphi-\varphi_{0}\right) g$, with $\varphi_{0}=\left.\frac{\partial u_{0}}{\partial \nu}\right|_{\partial \Omega}$. Moreover stronger smoothness assumptions on $D$ were made.

We wish to point out that the above estimates can also be employed when a portion $\Gamma$ of the boundary of $\Omega$ is not accessible to measurements (a very frequent situation in applications), since it is not necessary to measure the potential on $\Gamma$, provided $\varphi \equiv 0$ on such a portion of $\partial \Omega$.

Our approach to (1.3) is based on the following considerations. By the weak formulation of the Neumann problem (see Lemma 2.1 for details) we are led to the following inequalities

$$
\left(\frac{k-1}{k}\right) \int_{D}\left|\nabla u_{0}\right|^{2} \leq \delta \mathcal{W} \leq(k-1) \int_{D}\left|\nabla u_{0}\right|^{2},
$$

which were first derived in [KSS]. The left hand side of (1.3) is then obtained noticing that, by a standard interior gradient estimate,

$$
\int_{D}\left|\nabla u_{0}\right|^{2} \leq C|D|
$$

where $C$ depends on $\Omega, d_{0}$ and $\varphi$. In order to obtain the right hand side of (1.3), we notice that it is more troublesome to obtain the reverse inequality to (1.5)

$$
\int_{D}\left|\nabla u_{0}\right|^{2} \geq K|D|
$$

with a constant $K$ independent of $D$, since $\nabla u_{0}$ may vanish at interior points. This is readily understood by a simple example: set $n=2, \Omega=B_{1}(0), D=B_{r}(0), r<\frac{1}{2}$ 
and for some $j=2,3, \ldots, \varphi(x)=\sin j \theta$, with $(\rho, \theta)$ the polar coordinates. Then

$$
\int_{D}\left|\nabla u_{0}\right|^{2}=\frac{\pi}{j} r^{2 j}=O\left(|D|^{j}\right) .
$$

In Theorem 2.1, given $h_{1}>0$, we shall a priori assume on $D$ that

$$
\left|D_{h_{1}}\right| \geq \frac{1}{2}|D|
$$

where we denote $D_{h}=\{x \in D \mid \operatorname{dist}(x, \partial D)>h\}$.

The key argument of the proof will consist in showing that (1.6) holds with a constant $K$ also depending on $h_{1}$.

In Theorem 2.2 we instead consider the case when no a priori assumption is made on $D$. We have been able to treat this general case at the cost of allowing different growth rates for the upper and lower bounds on $|D|$. Namely we obtain

$$
C_{1} \frac{\delta \mathcal{W}}{\int_{\partial \Omega} g_{0} \varphi} \leq|D| \leq C_{2}\left(\frac{\delta \mathcal{W}}{\int_{\partial \Omega} g_{0} \varphi}\right)^{\frac{1}{p}}
$$

for some $p>1$. In fact, in this general case, the substitute to the estimate (1.6) is

$$
\int_{D}\left|\nabla u_{0}\right|^{2} \geq K|D|^{p}
$$

where $K, p$ depend on $\Omega, d_{0}, \varphi$. We observe that both estimates (1.6) and (1.9) will be derived from quantitative forms (Lemma 2.2 and Theorem G-L) of the unique continuation principle, essentially based on the results by Garofalo and Lin [GL], where they developed an elegant theory which connects the unique continuation property for solutions of elliptic partial differential equations with the theory of $A_{p}$ Muckenhoupt weights.

Finally, we point out that our technique also applies when the background conductivity in $\Omega$ and the one in $D$ are nonuniform and nonisotropic; in fact our theorems will be stated in this greater generality. We shall only require bounds on ellipticity, on the jump between the two conductivities, and on the Lipschitz continuity of the background conductivity in $\Omega$. See (2.1)-(2.3) below for a precise statement.

\section{The MAIN RESUlts}

Let $\Omega$ be a bounded domain in $\mathbb{R}^{n}$. Let $D \subset \Omega$ be measurable, $\operatorname{dist}(D, \partial \Omega) \geq d_{0}$, for some positive number $d_{0}$.

Let us represent by two symmetric $n \times n$ matrix valued functions in $\Omega, A=A(x)$, $B=B(x) \in L^{\infty}(\Omega)$, the conductivities in $\Omega \backslash \bar{D}$ and in $D$ respectively. On such conductivities we shall assume

(i) (uniform ellipticity for $A$ ) there exists $\lambda, 0<\lambda \leq 1$, such that:

$$
\lambda I d \leq A \leq \lambda^{-1} I d \quad \text { a.e. in } \Omega,
$$

(ii) (bound on the jump and uniform ellipticity for $B$ ) there exist $\mu, \eta>0$ such that either

$$
\eta A \leq B-A, \quad B \leq \mu A \quad \text { a.e. in } \Omega
$$

or

$$
\eta A \leq A-B, \quad \mu A \leq B \quad \text { a.e. in } \Omega
$$


(iii) (Lipschitz continuity for $A$ ) there exists $\Gamma>0$ such that for every $x, y \in \Omega$

$$
|A(x)-A(y)| \leq \Gamma|x-y| .
$$

Remarks. (i) Let us stress that no Lipschitz continuity is assumed on $B$.

(ii) When $(2.2+)$ holds it follows that $\mu>1$, whereas when (2.2-) holds it follows that $\mu<1$.

(iii) In the case of constant conductivities $A=I d, B=k I d, k \neq 1$, we have that $\lambda=1, \mu=k$ and $\eta=|k-1|$. Case $(2.2+)$ corresponds to the case $k>1$, whereas case $(2.2-)$ corresponds to the case $k<1$.

Given $\varphi \in L^{2}(\partial \Omega), \varphi \not \equiv 0$, such that $\int_{\partial \Omega} \varphi=0$, let $u, u_{0} \in H^{1}(\Omega)$ be the unique weak solutions to the Neumann problems

$$
\begin{aligned}
& \left\{\begin{array}{rlrl}
\operatorname{div}\left(\left(A \chi_{\Omega \backslash \bar{D}}+B \chi_{D}\right) \nabla u\right) & =0 & & \text { in } \Omega, \\
A D u \cdot \nu & =\varphi & & \text { on } \partial \Omega, \\
\int_{\Omega} u & =0, &
\end{array}\right.
\end{aligned}
$$

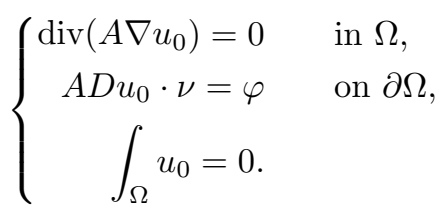

We let $g, g_{0} \in H^{1 / 2}(\partial \Omega)$ be the traces of $u, u_{0}$ respectively, on $\partial \Omega$.

Definition 2.1. We shall say that $\partial \Omega$ belongs to the class $C^{1,1}$, with constants $r_{0}$, $M_{0}$, if for any $x_{0} \in \partial \Omega, \partial \Omega \cap B_{r_{0}}\left(x_{0}\right)$ is a connected surface the equation of which, in a cartesian coordinate system having origin at $x_{0}$ and $x_{n}$-axis in the direction of the outer normal $\nu$ to $\Omega$ at $x_{0}$, is of the form $x_{n}=\Phi\left(x_{1}, \ldots, x_{n-1}\right)$, with $\Phi$ of class $C^{1,1}$ in the $(n-1)$-dimensional ball $B_{r_{0}}\left(x_{0}\right) \cap\left\{x_{n}=0\right\}, \Phi(0)=0, \nabla \Phi(0)=0$ and $\|\Phi\|_{C^{1,1}} \leq M_{0}$.

For any $h>0$, we shall denote

$$
D_{h}=\{x \in D \mid \operatorname{dist}(x, \partial D)>h\} .
$$

Theorem 2.1. Let $\Omega$ be a bounded domain in $\mathbb{R}^{n}$ such that $\partial \Omega \in C^{1,1}$ with constants $r_{0}, M_{0}$, and let $h_{1}>0$ be such that $\left|D_{h_{1}}\right| \geq \frac{1}{2}|D|$. Let $A, B$ satisfy (i)-(iii). If $(2.2+)$ holds, then we have

$$
\frac{1}{\mu-1} C_{1}^{+} \frac{\int_{\partial \Omega}\left(g_{0}-g\right) \varphi}{\int_{\partial \Omega} g_{0} \varphi} \leq|D| \leq \frac{\mu}{\eta} C_{2}^{+} \frac{\int_{\partial \Omega}\left(g_{0}-g\right) \varphi}{\int_{\partial \Omega} g_{0} \varphi} .
$$

If, conversely, (2.2-) holds, then we have

$$
\frac{\mu}{1-\mu} C_{1}^{-} \frac{\int_{\partial \Omega}\left(g-g_{0}\right) \varphi}{\int_{\partial \Omega} g_{0} \varphi} \leq|D| \leq \frac{1}{\eta} C_{2}^{-} \frac{\int_{\partial \Omega}\left(g-g_{0}\right) \varphi}{\int_{\partial \Omega} g_{0} \varphi},
$$

where $C_{1}^{+}, C_{1}^{-}$depend on $d_{0},|\Omega|, \lambda, \Gamma$ only, and $C_{2}^{+}, C_{2}^{-}$depend only on the same quantities and in addition on $r_{0}, M_{0}, h_{1},\|\varphi\|_{L^{2}(\partial \Omega)} /\|\varphi\|_{H^{-1 / 2}(\partial \Omega)}$. 
Theorem 2.2. Let $\Omega$ be as in Theorem 2.1 and let $D$ be any measurable subset of $\Omega_{d_{0}}$. Let $A, B$ satisfy (i)-(iii). If $(2.2+)$ holds, then we have

$$
\frac{1}{\mu-1} C_{1}^{+} \frac{\int_{\partial \Omega}\left(g_{0}-g\right) \varphi}{\int_{\partial \Omega} g_{0} \varphi} \leq|D| \leq\left(\frac{\mu}{\eta}\right)^{\frac{1}{p}} C_{2}^{+}\left(\frac{\int_{\partial \Omega}\left(g_{0}-g\right) \varphi}{\int_{\partial \Omega} g_{0} \varphi}\right)^{\frac{1}{p}} .
$$

If, conversely, (2.2-) holds, then we have

$$
\frac{\mu}{1-\mu} C_{1}^{-} \frac{\int_{\partial \Omega}\left(g-g_{0}\right) \varphi}{\int_{\partial \Omega} g_{0} \varphi} \leq|D| \leq\left(\frac{1}{\eta}\right)^{\frac{1}{p}} C_{2}^{-}\left(\frac{\int_{\partial \Omega}\left(g-g_{0}\right) \varphi}{\int_{\partial \Omega} g_{0} \varphi}\right)^{\frac{1}{p}}
$$

where $C_{1}^{+}, C_{1}^{-}$are the same as in Theorem 2.1 and $p>1, C_{2}^{+}, C_{2}^{-}$depend on $d_{0}$, $|\Omega|, \lambda, \Gamma, r_{0}, M_{0},\|\varphi\|_{L^{2}(\partial \Omega)} /\|\varphi\|_{H^{-1 / 2}(\partial \Omega)}$ only.

Remarks. (i) Observe that the quantity $h_{1}$ involves a sort of weak smoothness condition on $\partial D$. For instance, $h_{1}$ can be easily estimated when each connected component $D_{j}$ of $D$ has boundary $\partial D_{j}$ of the Lipschitz class with prescribed constants $r_{1}, M_{1}$, defined analogously to Definition 2.1 (see [AR, Lemma 2.8] for related estimates).

(ii) It will become apparent from the proof of Lemma 2.2 and from the Example below that the estimates on the right hand sides of $(2.6 \pm),(2.7 \pm)$ deteriorate as $\|\varphi\|_{L^{2}(\partial \Omega)} /\|\varphi\|_{H^{-1 / 2}(\partial \Omega)}$ increases. Such a ratio can be viewed as a parameter measuring the oscillation rate of $\varphi$ (see [AR, Concluding Remarks]). It is therefore convenient to choose, if possible, $\varphi$ slowly varying between its negative and positive values. For instance in [KSS], when $A=I d, B=k I d$, a suggested choice was $\varphi(x)=v \cdot \nu(x), v$ being a constant vector.

(iii) Let us observe in the above theorems that in order to determine $g_{0}$, we need the exact knowledge of the background conductivity $A$, whereas the conductivity $B$ (inside $D$ ) does not need to be precisely known; in fact only the bounds (ii) are required.

Let us show, by a simple example, that the estimates in Theorems 2.1 and 2.2 are nearly the best possible.

Example. Let us set $n=2, \Omega=B_{1}(0), D=B_{r}(0), 0<r \leq \frac{1}{2}, A=I d, B=k I d$, and, for the sake of definiteness, $k=2$. Let us pick $\varphi(x)=\sin j \theta$, for some $j=2,3, \ldots$, where we denote by $(\rho, \theta)$ the polar coordinates. In this case we have

$$
\begin{gathered}
|D|=\pi r^{2} \\
\frac{\delta \mathcal{W}}{\int_{\partial \Omega} g_{0} \varphi}=\frac{2 r^{2 j}}{3+r^{2 j}}=O\left(r^{2 j}\right), \quad \text { as } r \rightarrow 0 \\
\frac{\|\varphi\|_{L^{2}(\partial \Omega)}}{\|\varphi\|_{H^{-1 / 2}(\partial \Omega)}}=\sqrt{j}
\end{gathered}
$$

If the hypotheses of Theorem 2.1 are assumed, we must have

$$
r \geq \frac{\sqrt{2}}{\sqrt{2}-1} h_{1}
$$

and the constant on the right hand side of $(2.6+)$ must satisfy

$$
C_{2}^{+} \geq \frac{3 \pi}{4} \exp \left[-2\left(\frac{\|\varphi\|_{L^{2}(\partial \Omega)}^{2}}{\|\varphi\|_{H^{-1 / 2}(\partial \Omega)}^{2}}-1\right) \ln \left(\frac{\sqrt{2}}{\sqrt{2}-1} h_{1}\right)\right] .
$$


On the other hand, the constants $p, C_{2}^{+}$in the right hand side of $(2.7+)$ must satisfy

$$
p \geq \frac{\|\varphi\|_{L^{2}(\partial \Omega)}^{2}}{\|\varphi\|_{H^{-1 / 2}(\partial \Omega)}^{2}}, \quad C_{2}^{+} \geq \frac{\pi}{4} .
$$

Lemma 2.1. Let $\Omega$ be as in Theorem 2.1 and let $D \Subset \Omega$ be measurable. Let $A, B$ satisfy (i)-(iii). If (2.2+) holds, then we have

$$
\frac{\eta \lambda}{\mu} \int_{D}\left|\nabla u_{0}\right|^{2} \leq \int_{\partial \Omega}\left(g_{0}-g\right) \varphi \leq \frac{\mu-1}{\lambda} \int_{D}\left|\nabla u_{0}\right|^{2}
$$

if instead (2.2-) holds, then we have

$$
\eta \lambda \int_{D}\left|\nabla u_{0}\right|^{2} \leq \int_{\partial \Omega}\left(g-g_{0}\right) \varphi \leq \frac{1-\mu}{\lambda \mu} \int_{D}\left|\nabla u_{0}\right|^{2} .
$$

Proof. The proof is based on the following identities:

$\int_{\Omega}\left(A \chi_{\Omega \backslash \bar{D}}+B \chi_{D}\right) \nabla\left(u-u_{0}\right) \cdot \nabla\left(u-u_{0}\right)-\int_{D}(B-A) \nabla u_{0} \cdot \nabla u_{0}=\int_{\partial \Omega}\left(g-g_{0}\right) \varphi$,

$$
\begin{gathered}
\int_{\Omega} A \nabla\left(u-u_{0}\right) \cdot \nabla\left(u-u_{0}\right)+\int_{D}(B-A) \nabla u \cdot \nabla u=\int_{\partial \Omega}\left(g_{0}-g\right) \varphi \\
\int_{D}(A-B) \nabla u \cdot \nabla u_{0}=\int_{\partial \Omega}\left(g-g_{0}\right) \varphi
\end{gathered}
$$

which can be derived, similarly to (3.5)-(3.6) in [KSS], from the weak formulation of (2.4) and (2.5).

In case $(2.2+)$ holds, we have by $(2.9)$

$$
\int_{\partial \Omega}\left(g_{0}-g\right) \varphi \leq \int_{D}(B-A) \nabla u_{0} \cdot \nabla u_{0}
$$

Moreover for every $\epsilon>0$

$$
\begin{gathered}
\int_{D}(B-A) \nabla u_{0} \cdot \nabla u_{0} \\
\leq(1+\epsilon) \int_{D}(B-A) \nabla\left(u-u_{0}\right) \cdot \nabla\left(u-u_{0}\right)+\left(1+\frac{1}{\epsilon}\right) \int_{D}(B-A) \nabla u \cdot \nabla u \\
\leq(1+\epsilon)(\mu-1)\left[\int_{D} A \nabla\left(u-u_{0}\right) \cdot \nabla\left(u-u_{0}\right)+\frac{1}{\epsilon(\mu-1)} \int_{D}(B-A) \nabla u \cdot \nabla u\right],
\end{gathered}
$$

which, choosing $\epsilon=\frac{1}{\mu-1}$ and using (2.10), is dominated by $\mu \int_{\partial \Omega}\left(g_{0}-g\right) \varphi$, so that

$$
\int_{D}(B-A) \nabla u_{0} \cdot \nabla u_{0} \leq \mu \int_{\partial \Omega}\left(g_{0}-g\right) \varphi .
$$

In case $(2.2-)$ holds, we have by $(2.9)$

$$
\int_{\partial \Omega}\left(g-g_{0}\right) \varphi \geq \int_{D}(A-B) \nabla u_{0} \cdot \nabla u_{0} .
$$


Now, using (2.11), for every $\epsilon>0$ we obtain

$$
\begin{aligned}
\int_{\partial \Omega}\left(g-g_{0}\right) \varphi & =\int_{D}(A-B) \nabla u \cdot \nabla u_{0} \\
& \leq \frac{\epsilon}{2} \int_{D}(A-B) \nabla u \cdot \nabla u+\frac{1}{2 \epsilon} \int_{D}(A-B) \nabla u_{0} \cdot \nabla u_{0} .
\end{aligned}
$$

On the other hand, by (2.10) and (2.9)

$$
\begin{gathered}
\int_{D}(A-B) \nabla u \cdot \nabla u=\int_{\partial \Omega}\left(g-g_{0}\right) \varphi+\int_{\Omega} A \nabla\left(u-u_{0}\right) \cdot \nabla\left(u-u_{0}\right) \\
\leq \int_{\partial \Omega}\left(g-g_{0}\right) \varphi+\frac{1}{\mu} \int_{\Omega}\left(A \chi_{\Omega \backslash \bar{D}}+B \chi_{D}\right) \nabla\left(u-u_{0}\right) \cdot \nabla\left(u-u_{0}\right) \\
=\frac{\mu+1}{\mu} \int_{\partial \Omega}\left(g-g_{0}\right) \varphi-\frac{1}{\mu} \int_{D}(A-B) \nabla u_{0} \cdot \nabla u_{0} .
\end{gathered}
$$

By inserting inequality (2.13) in (2.12) we have that, for every $0<\epsilon<\frac{2 \mu}{\mu+1}$,

$$
\int_{\partial \Omega}\left(g-g_{0}\right) \varphi \leq \alpha(\epsilon) \int_{D}(A-B) \nabla u_{0} \cdot \nabla u_{0}
$$

where

$$
\alpha(\epsilon)=\frac{\mu-\epsilon^{2}}{\epsilon(2 \mu-\epsilon \mu-\epsilon)} .
$$

The minimum of $\alpha(\epsilon)$ occurs when $\epsilon=\mu$, and in this case we deduce

$$
\int_{\partial \Omega}\left(g-g_{0}\right) \varphi \leq \frac{1}{\mu} \int_{D}(A-B) \nabla u_{0} \cdot \nabla u_{0} .
$$

Therefore, if $(2.2+)$ holds, we have that

$$
\frac{1}{\mu} \int_{D}(B-A) \nabla u_{0} \cdot \nabla u_{0} \leq \int_{\partial \Omega}\left(g_{0}-g\right) \varphi \leq \int_{D}(B-A) \nabla u_{0} \cdot \nabla u_{0},
$$

whereas, if $(2.2-)$ holds, we have that

$$
\int_{D}(A-B) \nabla u_{0} \cdot \nabla u_{0} \leq \int_{\partial \Omega}\left(g-g_{0}\right) \varphi \leq \frac{1}{\mu} \int_{D}(A-B) \nabla u_{0} \cdot \nabla u_{0} .
$$

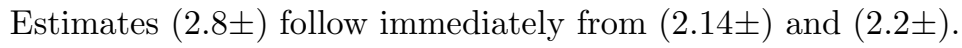

Lemma 2.2. Let $\Omega$ be a bounded domain in $\mathbb{R}^{n}$, such that $\partial \Omega \in C^{1,1}$ with constants $r_{0}, M_{0}$. Let A satisfy (i), (iii). For every $x \in \Omega_{4 \rho}$ and for every $\rho>0$, we have

$$
\int_{B_{\rho}(x)}\left|\nabla u_{0}\right|^{2} \geq C_{\rho} \int_{\Omega}\left|\nabla u_{0}\right|^{2}
$$

where $C_{\rho}$ depends on $\lambda, \Gamma,|\Omega|, r_{0}, M_{0},\|\varphi\|_{L^{2}(\partial \Omega)} /\|\varphi\|_{H^{-1 / 2}(\partial \Omega)}$ and $\rho$ only.

Proof. Let $\rho_{0}$, depending on $r_{0}, M_{0}$ only, be such that $\Omega_{4 \rho}$ is connected for every $\rho \leq \rho_{0}$. Without loss of generality, we may assume, for this proof, $\rho \leq \rho_{0}$. Given any $y \in \Omega_{4 \rho}$, let $\gamma$ be an arc in $\Omega_{4 \rho}$ joining $x$ and $y$. Let us define $\left\{x_{i}\right\}, i=1, \ldots, L$, as follows: $x_{1}=x, x_{i+1}=\gamma\left(t_{i}\right)$, where $t_{i}=\max \left\{t\right.$ s.t. $\left.\left|\gamma(t)-x_{i}\right|=2 \rho\right\}$ if $\left|x_{i}-y\right|>2 \rho$; otherwise let $i=L$ and stop the process. Then, by construction, the balls $B_{\rho}\left(x_{i}\right)$ are pairwise disjoint, $\left|x_{i+1}-x_{i}\right|=2 \rho$ for $i=1, \ldots, L-1,\left|x_{L}-y\right| \leq 2 \rho$.

At this stage we shall make use of a three sphere inequality for solutions $v$ to the elliptic equation in divergence form with Lipschitz coefficients. More precisely, 
for any $r^{*}>0$ there exist $C \geq 1, \delta \in(0,1)$ depending only on $\lambda, \Gamma$ and $r^{*}$, such that for every $x$ satisfying $B_{4 r^{*}}(x) \subset \Omega$ and for every $r \leq r^{*}$ we have

$$
\|v\|_{L^{2}\left(B_{7 r / 2}(x)\right)} \leq C\|v\|_{L^{2}\left(B_{r}(x)\right)}^{\delta}\|v\|_{L^{2}\left(B_{4 r}(x)\right)}^{1-\delta} .
$$

This result can be derived, through minor adaptations, from the estimates found by Garofalo and Lin in their proof of the unique continuation properties for this type of equations [GL]. In particular, choosing $v=u_{0}-\bar{c}$, with $\bar{c}=\left(\frac{1}{\left|B_{r}(x)\right|}\right) \int_{B_{r}(x)} u_{0}$, and applying Caccioppoli and Poincaré inequalities, we have

$$
\left\|\nabla u_{0}\right\|_{L^{2}\left(B_{3 r}(x)\right)} \leq C\left\|\nabla u_{0}\right\|_{L^{2}\left(B_{r}(x)\right)}^{\delta}\left\|\nabla u_{0}\right\|_{L^{2}\left(B_{4 r}(x)\right)}^{1-\delta} .
$$

Let us consider (2.16) for $x=x_{i}$ and $r=\rho$. We have

$$
\frac{\left\|\nabla u_{0}\right\|_{L^{2}\left(B_{\rho}\left(x_{i+1}\right)\right)}}{\left\|\nabla u_{0}\right\|_{L^{2}(\Omega)}} \leq C\left(\frac{\left\|\nabla u_{0}\right\|_{L^{2}\left(B_{\rho}\left(x_{i}\right)\right)}}{\left\|\nabla u_{0}\right\|_{L^{2}(\Omega)}}\right)^{\delta}
$$

for $i=1, \ldots, L-1$. By induction we have

$$
\frac{\left\|\nabla u_{0}\right\|_{L^{2}\left(B_{\rho}(y)\right)}}{\left\|\nabla u_{0}\right\|_{L^{2}(\Omega)}} \leq C^{1 /(1-\delta)}\left(\frac{\left\|\nabla u_{0}\right\|_{L^{2}\left(B_{\rho}(x)\right)}}{\left\|\nabla u_{0}\right\|_{L^{2}(\Omega)}}\right)^{\delta^{L}}
$$

where $C$ depends only on $\lambda, \Gamma$ and $L \leq \frac{|\Omega|}{\omega_{n} \rho^{n}}$. Let us cover $\Omega_{5 \rho}$ with internally nonoverlapping closed cubes of side $l=2 \rho / \sqrt{n}$. The number of such cubes is controlled by $N=\frac{|\Omega| n^{n / 2}}{2^{n} \rho^{n}}$. Therefore, from (2.18) we have

$$
\frac{\left\|\nabla u_{0}\right\|_{L^{2}\left(\Omega_{5 \rho}\right)}}{\left\|\nabla u_{0}\right\|_{L^{2}(\Omega)}} \leq C\left(\frac{\left\|\nabla u_{0}\right\|_{L^{2}\left(B_{\rho}(x)\right)}}{\left\|\nabla u_{0}\right\|_{L^{2}(\Omega)}}\right)^{\delta^{L}}
$$

where $C$ depends only on $\lambda, \Gamma,|\Omega|, \rho$.

In order to estimate from below the left hand side in (2.19) we set

$$
\frac{\left\|\nabla u_{0}\right\|_{L^{2}\left(\Omega_{5 \rho}\right)}^{2}}{\left\|\nabla u_{0}\right\|_{L^{2}(\Omega)}^{2}}=1-\frac{\int_{\Omega \backslash \Omega_{5 \rho}}\left|\nabla u_{0}\right|^{2}}{\int_{\Omega}\left|\nabla u_{0}\right|^{2}}
$$

On one hand we have:

Claim.

$$
\int_{\Omega \backslash \Omega_{5 \rho}}\left|\nabla u_{0}\right|^{2} \leq C \rho^{1 / n}\|\varphi\|_{L^{2}(\partial \Omega)}^{2}
$$

where $C$ depends only on $\lambda, \Gamma, r_{0}, M_{0},|\Omega|$.

Proof of the Claim. By Hölder's inequality

$$
\left\|\nabla u_{0}\right\|_{L^{2}\left(\Omega \backslash \Omega_{5 \rho}\right)}^{2} \leq\left|\Omega \backslash \Omega_{5 \rho}\right|^{1 / n}\left\|\nabla u_{0}\right\|_{L^{2 n /(n-1)}\left(\Omega \backslash \Omega_{5 \rho}\right)}^{2},
$$

and by Sobolev inequality (see, e.g., $[\mathrm{Ad}]$ )

$$
\left\|\nabla u_{0}\right\|_{L^{2 n /(n-1)}(\Omega)}^{2} \leq C\left\|\nabla u_{0}\right\|_{H^{1 / 2}(\Omega)}^{2},
$$

we have

$$
\left\|\nabla u_{0}\right\|_{L^{2}\left(\Omega \backslash \Omega_{5 \rho}\right)}^{2} \leq C\left|\Omega \backslash \Omega_{5 \rho}\right|^{1 / n}\left\|u_{0}\right\|_{H^{3 / 2}(\Omega)}^{2},
$$

where $C$ depends only on $r_{0}, M_{0},|\Omega|$.

Moreover, we have

$$
\left\|u_{0}\right\|_{H^{3 / 2}(\Omega)} \leq C\|\varphi\|_{L^{2}(\partial \Omega)}
$$


where $C$ depends only on $\lambda, \Gamma, r_{0}, M_{0},|\Omega|$. Inequality (2.24) follows, by interpolation (see $[\mathrm{LM}]$ ), from the trivial estimate

$$
\left\|u_{0}\right\|_{H^{1}(\Omega)} \leq C\|\varphi\|_{H^{-1 / 2}(\partial \Omega)},
$$

where $C$ depends only on $\lambda, r_{0}, M_{0},|\Omega|$, and from the elliptic estimate for the Neumann problem (see $[\mathrm{Gr}]$ )

$$
\left\|u_{0}\right\|_{H^{2}(\Omega)} \leq C\|\varphi\|_{H^{1 / 2}(\partial \Omega)}
$$

where $C$ depends only on $\lambda, \Gamma, r_{0}, M_{0},|\Omega|$.

Moreover,

$$
\left|\Omega \backslash \Omega_{5 \rho}\right| \leq C \rho,
$$

where $C$ depends only on $r_{0}, M_{0},|\Omega|$ (see (A.3) in [AR] for details). From (2.23), (2.24) and (2.27), the Claim follows.

On the other hand, we have the standard estimate

$$
\|\varphi\|_{H^{-1 / 2}(\partial \Omega)}^{2} \leq C \int_{\Omega}\left|\nabla u_{0}\right|^{2},
$$

where $C$ depends only on $\lambda, r_{0}, M_{0},|\Omega|$ and hence there exists $\bar{\rho}>0$ depending only on $\lambda, \Gamma,|\Omega|, r_{0}, M_{0},\|\varphi\|_{L^{2}(\partial \Omega)} /\|\varphi\|_{H^{-1 / 2}(\partial \Omega)}$ such that

$$
\frac{\int_{\Omega_{5 \rho}}\left|\nabla u_{0}\right|^{2}}{\int_{\Omega}\left|\nabla u_{0}\right|^{2}} \geq \frac{1}{2}
$$

for every $\rho, 0<\rho \leq \bar{\rho}$. Finally, from (2.19) and (2.29) the thesis follows when $0<\rho \leq \bar{\rho}$; for larger values of $\rho$, inequality (2.15) is then elementary.

Proof of Theorem 2.1. Let us consider the intermediate domain $\Omega_{d_{0} / 2}$. Recalling that $\operatorname{dist}(D, \partial \Omega) \geq d_{0}$, we have $\operatorname{dist}\left(D, \partial \Omega_{d_{0} / 2}\right) \geq \frac{d_{0}}{2}$. By standard elliptic estimates we have

$$
\sup _{D}\left|\nabla u_{0}\right| \leq C \sup _{\Omega_{d_{0} / 2}}\left|u_{0}\right| \leq C\left\|u_{0}\right\|_{L^{2}(\Omega)},
$$

where the constants depend on $\lambda, \Gamma, d_{0}$. From the trivial estimate

$$
\int_{\Omega}\left|\nabla u_{0}\right|^{2} \leq \lambda^{-1} \int_{\Omega} A \nabla u_{0} \cdot \nabla u_{0}=\lambda^{-1} \int_{\partial \Omega} g_{0} \varphi
$$

and from the Poincaré inequality, we have

$$
\sup _{D}\left|\nabla u_{0}\right| \leq C\left(\int_{\partial \Omega} g_{0} \varphi\right)^{\frac{1}{2}}
$$

where $C$ depends only on $\lambda, \Gamma, d_{0},|\Omega|$. The lower bound for $|D|$ in (2.6 \pm ) follows from the right hand side of $(2.8 \pm)$ and (2.30).

Next we prove the upper bound in (2.6 \pm$)$.

Let $\epsilon=\min \left(\frac{d_{0}}{2}, \frac{h_{1}}{\sqrt{n}}\right)$. Let us cover $D_{h_{1}}$ with internally nonoverlapping closed cubes $Q_{l}$ of side $\epsilon$, for $l=1, \ldots, L$. By the choice of $\epsilon$, the cubes $Q_{l}$ are contained in $D$. Hence

$$
\int_{D}\left|\nabla u_{0}\right|^{2} \geq \int_{\bigcup_{l=1}^{L} Q_{l}}\left|\nabla u_{0}\right|^{2} \geq \frac{\left|D_{h_{1}}\right|}{\epsilon^{n}} \int_{Q_{\bar{l}}}\left|\nabla u_{0}\right|^{2}
$$


where $\bar{l}$ is chosen in such a way that $\int_{Q_{\bar{l}}}\left|\nabla u_{0}\right|^{2}=\min _{l} \int_{Q_{l}}\left|\nabla u_{0}\right|^{2}$. Let $\bar{x}$ be the center of $Q_{\bar{l}}$. From (2.31), the estimate (2.15) in Lemma 2.2 with $x=\bar{x}, \rho=\frac{\epsilon}{2}$, and from the trivial estimate

$$
\int_{\Omega}\left|\nabla u_{0}\right|^{2} \geq \lambda \int_{\Omega} A \nabla u_{0} \cdot \nabla u_{0}=\lambda \int_{\partial \Omega} g_{0} \varphi
$$

we have

$$
\int_{D}\left|\nabla u_{0}\right|^{2} \geq\left(K \int_{\partial \Omega} g_{0} \varphi\right)|D|
$$

where $K$ depends only on $\lambda, \Gamma, d_{0},|\Omega|, r_{0}, M_{0}, h_{1},\|\varphi\|_{L^{2}(\partial \Omega)} /\|\varphi\|_{H^{-1 / 2}(\partial \Omega)}$. The

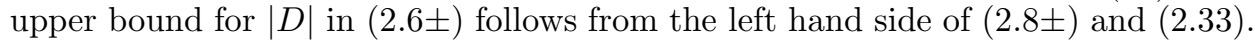

Before proceeding to the proof of Theorem 2.2, we note that only the upper estimates in $(2.7 \pm)$ are in need of a new proof, since the lower estimates in Theorem 2.1 also apply under the hypotheses of Theorem 2.2. For the proof of the upper estimates without assuming a priori information on $D$, we shall take advantage of the fact that $\left|\nabla u_{0}\right|^{2}$ is a Muckenhoupt weight (see [GL]).

Theorem G-L. Let $u_{0}$ be the solution to (2.5). For any $\bar{r}>0$ there exist $B>0$ and $p>1$ such that we have

$$
\left(\frac{1}{\left|B_{r}\right|} \int_{B_{r}}\left|\nabla u_{0}\right|^{2}\right)\left(\frac{1}{\left|B_{r}\right|} \int_{B_{r}}\left|\nabla u_{0}\right|^{-\frac{2}{p-1}}\right)^{p-1} \leq B
$$

for any ball $B_{r}$ such that $B_{4 r} \subset \Omega_{\bar{r}}$. Here $B, p$ depend only on $\lambda, \Gamma,|\Omega|, r_{0}, M_{0}$, $\bar{r}$ and $\|\varphi\|_{L^{2}(\partial \Omega)} /\|\varphi\|_{H^{-1 / 2}(\partial \Omega)}$.

Proof. This result stems from [GL, Theorem 1.1] by rather straightforward arguments in the style of the proof of Lemma 2.2 above.

Proof of Theorem 2.2. Let us pick $\bar{r}=\frac{d_{0}}{2}$ and $\epsilon=\frac{d_{0}}{5 \sqrt{n}}$. Let us cover $D$ with internally nonoverlapping closed cubes $Q_{j}, j=1, \ldots, J$, with side $\epsilon$. By Hölder's inequality, we have

$$
|D|=\int_{D}\left|\nabla u_{0}\right|^{-2 / p}\left|\nabla u_{0}\right|^{2 / p} \leq\left(\int_{\bigcup_{j=1}^{J} Q_{j}}\left|\nabla u_{0}\right|^{-2 /(p-1)}\right)^{(p-1) / p}\left(\int_{D}\left|\nabla u_{0}\right|^{2}\right)^{1 / p} .
$$

By using (2.34) on the balls $B_{j}$ circumscribing each cube $Q_{j}, j=1, \ldots, J$, we have

$$
\begin{aligned}
& \left(\int_{\bigcup_{j=1}^{J} Q_{j}}\left|\nabla u_{0}\right|^{-2 /(p-1)}\right)^{(p-1) / p}=\left(\epsilon^{n} \sum_{j=1}^{J} \frac{1}{\left|Q_{j}\right|} \int_{Q_{j}}\left|\nabla u_{0}\right|^{-2 /(p-1)}\right)^{(p-1) / p} \\
& \leq\left(\epsilon^{n} \sum_{j=1}^{J}\left(\frac{B[C(n)]^{p}}{\frac{1}{\left|Q_{j}\right|} \int_{Q_{j}}\left|\nabla u_{0}\right|^{2}}\right)^{1 /(p-1)}\right)^{(p-1) / p} \leq \frac{\left(J \epsilon^{n}\right)^{(p-1) / p} B^{1 / p} C(n)}{\min _{j}\left(\frac{1}{\left|Q_{j}\right|} \int_{Q_{j}}\left|\nabla u_{0}\right|^{2}\right)^{1 / p}},
\end{aligned}
$$


where $C(n)=\omega_{n}\left(\frac{\sqrt{n}}{2}\right)^{n}$. Now $J \epsilon^{n}=\sum_{j=1}^{J}\left|Q_{j}\right| \leq|\Omega|$ and hence

$$
|D| \leq|\Omega|^{(p-1) / p}(B)^{1 / p} C(n)\left(\frac{\epsilon^{n} \int_{D}\left|\nabla u_{0}\right|^{2}}{\min _{j} \int_{Q_{j}}\left|\nabla u_{0}\right|^{2}}\right)^{1 / p} .
$$

By (2.15), (2.32) and (2.36) we have

$$
\int_{D}\left|\nabla u_{0}\right|^{2} \geq\left(K \int_{\partial \Omega} g_{0} \varphi\right)|D|^{p}
$$

where $K$ depends only on $\lambda, \Gamma, d_{0},|\Omega|, r_{0}, M_{0},\|\varphi\|_{L^{2}(\partial \Omega)} /\|\varphi\|_{H^{-1 / 2}(\partial \Omega)}$. The right hand side of (2.7 \pm ) follows from the left hand side of (2.8 \pm ) and (2.37).

Remark. We observe that the results of Theorems 2.1 and 2.2 also remain valid when $\partial \Omega$ is merely Lipschitz. In fact, the only stage at which the smoothness of $\partial \Omega$ has been used is in (2.20), and indeed such estimate could be improved as follows:

$$
\int_{\Omega \backslash \Omega_{5 \rho}}\left|\nabla u_{0}\right|^{2} \leq C \rho\|\varphi\|_{L^{2}(\partial \Omega)}^{2}
$$

where $C>0$ depends only on $\lambda, \Gamma$ and on the Lipschitz character of $\Omega$.

This last estimate (2.38) could be derived by a rather lengthy adaptation of deep results by Kenig and Pipher $[\mathrm{KP}]$; see also $[\mathrm{K}]$ and in particular Theorems 1.8.13, 2.4.5 and 2.8.5.

\section{REFERENCES}

[Ad] R. A. Adams, Sobolev Spaces, Academic Press, New York, 1975. MR 56:9247

[AI] G. Alessandrini and V. Isakov, Analyticity and uniqueness for the inverse conductivity problem, Rend. Ist. Mat. Univ. Trieste 28 (I/II) (1996), 351-370. MR 98g:35218

[AIP] G. Alessandrini, V. Isakov, and J. Powell, Local uniqueness in the inverse conductivity problem with one measurement, Trans. Amer. Math. Soc. 347 (1995), 3031-3041. MR 95m:35197

[Al] G. Alessandrini, Remark on a paper by Bellout and Friedman,, Boll. Un. Mat. Ital. A 23 (1989), 243-249. MR 90g:31005

[AR] G. Alessandrini and E. Rosset, The inverse conductivity problem with one measurement: bounds on the size of the unknown object, SIAM J. Appl. Math. 58, 4 (1998), 1060-1071. CMP 98:12

[BF] H. Bellout and A. Friedman, Identification problems in potential theory, Arch. Rational Mech. Anal. 101 (1988), 143-160. MR 90g:31005

[BFI] H. Bellout, A. Friedman, and V. Isakov, Stability for an inverse problem in potential theory, Trans. Amer. Math. Soc. 332 (1992), 271-296. MR 92j:31010

[BFS] B. Barceló, E. Fabes, and J. K. Seo, The inverse conductivity problem with one measurement: uniqueness for convex polyhedra, Proc. Amer. Math. Soc. 122 (1994), 183-189. MR 94k:35320

[Br] K. Bryan, Single measurement detection of a discontinuous conductivity, Comm. Partial Differential Equations 15 (1990), 503-514. MR 91e:78015

[C] V. G. Cherednichenko, A problem in the conjugation of harmonic functions and its inverse, Differential Equations 18 (1982), 682-689, 734. MR 83h:31002

[F] A. Friedman, Detection of mines by electric measurements, SIAM J. Appl. Math. 47 (1987), 201-212. MR 88c:35149

[FI] A. Friedman and V. Isakov, On the uniqueness in the inverse conductivity problem with one measurement, Indiana Univ. Math. J. 38 (1989), 563-579. MR 91a:35164

[GL] N. Garofalo and F. Lin, Monotonicity properties of variational integrals, $A_{p}$ weights and unique continuation, Indiana Univ. Math. J. 35 (1986), 245-268. MR 88b:35059

[Gr] P. Grisvard, Elliptic problems in nonsmooth domains, Monographs and Studies in Mathematics 24, Pitman, Boston, 1985. MR 86m:35044 
[IP] V. Isakov and J. Powell, On the inverse conductivity problem with one measurement, Inverse Problems 6 (1990), 311-318. MR 91e:35212a; corrigendum MR 91e:335212b

[K] C. E. Kenig, Harmonic Analysis Techniques for Second Order Elliptic Boundary Value Problems, American Mathematical Society, Providence R. I., 1994. MR 96a:35040

[KP] C. E. Kenig and J. Pipher, The Neumann problem for elliptic equations with non-smooth coefficients, Invent. Math. 113 (1993), 447-509. MR 95b:35046

[KSS] H. Kang, J. K. Seo, and D. Sheen, The inverse conductivity problem with one measurement: stability and estimation of size, SIAM J. Math. Anal. 28 (1997), 1389-1405. MR 98k:86021

[LM] J. L. Lions and E. Magenes, Non-homogeneous boundary value problems and applications I, Springer-Verlag, Berlin, 1972. MR 50:2670

[P] J. Powell, On a small perturbation in the two dimensional inverse conductivity problem, J. Math. Anal. Appl. 175 (1993), 292-304. MR 94c:35169

[S] J. K. Seo, A uniqueness result on inverse conductivity problem with two measurements, J. Fourier Anal. Appl. 2 (1996), 227-235. MR 97b:35196

Dipartimento di Scienze Matematiche, Università Degli Studi di Trieste, 34100 TriESTE, ITALY

E-mail address: alessang@univ.trieste.it

E-mail address: rossedi@univ.trieste.it

Department of Mathematics, Yonsey University, Seoul 120-749, Korea

E-mail address: seoj@bubble.yonsei.ac.kr 